\title{
UPLC-QTOF (ESI +) MS Analysis Applied for Targeted Profiling of Human Urine Amino Acids
}

\author{
Florina ROMANCIUC*1), Raluca M. POP ${ }^{2,3)}$, Carmen SOCACIU ${ }^{1,2)}$ \\ 1)University of Agricultural Sciences and Veterinary Medicine, 3-5 Mănăștur Street, Cluj-Napoca, \\ Romania \\ ${ }^{2)}$ Center for Applied Biotechnology CCD-BIODIATEC Cluj-Napoca, Romania \\ ${ }^{3)}$ University of Medicine and Pharmacy "Iuliu Hatieganu" Cluj-Napoca, 8 Victor Babes Street, Cluj- \\ Napoca, Romania \\ *Corresponding author, email: florina_romanciuc@yahoo.com
}

Bulletin UASVM Animal Science and Biotechnologies 71(2) / 2014,

Print ISSN 1843-5262; Electronic ISSN 1843-536X

DOI:10.15835/buasvmcn-asb:10810

\begin{abstract}
Amino acids, the building blocks of proteins are providing fundamental information about metabolic status and early disease diagnosis. For an accurate interpretation of metabolic status, amino acid reference values for healthy humans are required. Most studies have pointed out that, the most accurate, reliable and sensitive tool to evaluate amino acids in biofluids (blood or urine) is liquid chromatography coupled with mass spectrometry (LC/MS). The aim of this study was to determine and optimize the urine amino acids profile by LC-QTOF (ESI+) MS, comparatively, using two generic samples from a healthy male and female. Urine aminoacids were purified using solid phase extraction, a pre-column derivatization and liquid/liquid extraction according to Ez-Faast kit (Phenomenex). The derivatized samples were analyzed by LC-QTOF-(ESI+)-MS and data were processed by Data Analysis-Extracted Ion Chromatogram software. A number of 16 amino acids were found in both samples, the male urine showed higher values for 10 amino acids (hydroxyproline, alanine, sarcosine, lysine, histidine, tryptophan, leucine, phenylalanine, cysteine and tyrosine), while glutamine and valine were higher in female urine. Most of the amino acids identified were essential amino acids found in both cases like: glutamine, proline, lysine, histidine, valine, tryptophan, leucine and phenylalanine. The main differences between the male vs female aminoacid fingerprints of urine were mainly quantitative and not qualitative. The amino acid concentrations ranged from $1.48 \mathrm{nmol} / \mathrm{mg}$ creatinine (proline) to $203.13 \mathrm{nmol} / \mathrm{mg}$ (histidine). The concentration of amino acids in man were 2 times higher than for female, while the percentages of essential amino acids were similar in both gender.
\end{abstract}

Keywords: amino acids, urine LC-QTOF/MS, pre-column derivatization, targeted metabolomics

\section{INTRODUCTION}

Amino acids, the building units of proteins, play an essential physiological role as metabolic precursors, regulators and intermediate metabolites, therefore they can potentially serve as indicators of the overall physiological status (Miyagi et al., 2011) or specific biomarkers of different pathological. Of those 20 amino acids found in proteins, eleven can be synthesized in mammalian cells called non-essential and the other nine are obtained from extracellular sources named essential amino acids (Bhutia et al., 2014). Amino acid concentrations in plasma and urine, especially the essential amino acids may vary considerably depending on diet, geographic area, age and sex so they have a personalized profile (Pitkanen et al., 2003).

Since the metabolic amino-acid balance changes in patients with various diseases, metabolomic fingerprint and quantitative prodile may act as an indicator of the altered physiological status (Hassan, 2012; Shen, 2013). Several investigations have pointed out that changes in the level of some amino acids may be considered as risk factor for developing certain diseases such as cardiovascular diseases (Tang et al., 2009), diabetes (Wang et al., 2013), liver diseases (Baniasadi et al., 2013). 
Moreover, there are several research studies focused on the amino acids profile changes in relation to different types of cancer diagnosis. Consequently, in patients with lung and breast cancer, tryptophan concentration has been correlated with tumor presence (Lai et al., 2005) while low levels of plasma arginine were correlated with breast and colorectal cancer (Okamoto, 2009; Visser, 2005). Also, it was reported that modified levels of amino acids in urine may be useful in ovarian and breast cancer detection at early stages (Slupsky et al., 2010). Kami et al. (2013) showed that the correlation between different metabolites and amino acid profile can distinguish between normal and tumor tissue in patients with prostate and lung cancer. They found that leucine, isoleucine and valine were significantly increased in either cases (Kami et al., 2013).

Generally, two types of amino acid profile alterations were observed in cancer, those reflecting metabolic changes common to many cancers, which are a limited to a set of amino acids, and those specific to each type of cancer which concerns a larger group of amino acids (Miyagi et al., 2011). In these sense, metabolomics, both targeted and untargeted has a great potential to quantify biomarkers of interest from tissue or biofluides and to perform validation and implementation in clinical practice. There are two major techniques that are most commonly used in focused or open profiling methods for metabolites evaluation: nuclear magnetic resonance (NMR) and liquid chromatography coupled with mass spectrometry (LC/MS). The last one is the most accurate and reliable technique that can detect small metabolites that cannot be measured by other methods (Kasture et al., 2012)

Many various techniques have been design for amino acids detection with a wide range of reagents that imply a very laborious sample preparation with protein precipitation, extraction and derivatization that are specially meant for LC/MS application (Meesters et al., 2009). Liquid chromatography coupled with mass spectrometry (LC/MS) is one of the most widely used method for detection and quantification for amino acids (Grebe and Singh, 2011).

Therefore this study aims to apply targeted metabolomics on an healthy male and female urine for determination of amino acids profile by LCQTOF (ESI+) MS using a pre-column derivatization protocol. To reduce sample preparation and analysis time the amino acid profile of aliphatic and aromatic amino acids were determined using an commercially available kit from Phenomenex (EZ:faast ${ }^{\mathrm{TM}}$ amino acids analysis kit). The short time and relatively mild derivatization protocol, along with the large number of amino acids and dipeptides with detection limits about $1 \mu \mathrm{M}$ for most of the amino acids, makes the Phenomenex EZ:faast ${ }^{\mathrm{TM}}$ amino acids LC/MS free (physiological) amino acid analysis kit suitable for stable isotope trace studies (Wilson et al., 2011).

\section{MATERIALS AND METHODS}

Urine sample collection. Urine samples were obtained from two healthy people, one male of 51 and one female of 44 years of age, in order to determine the similarities and differences on amino acid profile between the genders. From each volunteer $10 \mathrm{ml}$ of normal spot urine were collected in sterile urine containers. Homogenized samples were purified by 5 minutes centrifugation at 2000 revolutions per minute (RPM). After centrifugation the supernatant was aliquoted and stored at $-20^{\circ} \mathrm{C}$ until analysis.

Extraction and derivatization of free amino acids. Urine sample preparation for UPLC-QTOF(ESI+)-MS analysis was carried out by using the EZ:faast amino acid free (physiological) analysis kit. The EZ:faast ${ }^{\mathrm{TM}}$ amino acids analysis kit from Phenomenex procedure consist of a solid phase extraction (SPE) step followed by a derivatization and a liquid/liquid extraction step. $100 \mu \mathrm{l}$ of thawed urine was pipetted into a glass sample preparation vial and then $100 \mu \mathrm{l}$ of internal standard was added. The diluted sample was pulled slowly through a solid phase extraction tip by moving the piston of a a $1.5 \mathrm{ml}$ syringe attached to the sorbent tip. The internal standard solution included homoarginine (HARG), methionine- $\mathrm{D}_{3}$ (MET- $\mathrm{D}_{3}$ ) and homophenylalanine (HPHE) that are not naturally found in the samples. Then, 200 $\mu \mathrm{l}$ of washing solution (N-propanol) was added into the glass vial and slowly pulled through the SPE tip to remove urea and other components found in urine. The sorbent tip was detached into the vial and the liquid accumulated in the syringe was discarded. The next step was to discard the sample retained on the SPE tip by adding in the sample preparation vial $200 \mu$ l eluting/extraction solution consisting on a mix of reagent 1 (sodium 
hydroxide) and reagent 2 (N-propanol, 3-picoline) (solution A/B: 3/2\% (v/v)), next the SPE tip was attach to a $0.6 \mathrm{ml}$ syringe and wet with the elution medium. The solid phase sorbent particles ware then expelled into the sample preparation vial by pushing the piston down. Derivatization agent was added (50 $\mu \mathrm{l}$ propyl chloroformate), was vortexed $10 \mathrm{~s}$ and let for $1 \mathrm{~min}$. to react. Above 100 $\mu \mathrm{l}$ of organic extraction solvent was added and the reaction procedure was repeated. The part of the upper layer was transferred into an autosampler vial for LC injection.

UPLC-QTOF (ESI+) MS analysis. UPLC-QTOF(ESI+)-MS analysis was performed using a Thermo Scientific HPLC UltiMate 3000 system equipped with a quaternary pump delivery system Dionex and MS detection by a Bruker Daltonics MaXis Impact device. The column used for separation in LC/MS analysis was an EZ:faast AAA-MS 250x3.0 $\mathrm{mm}$ i.d. column from Phenomenex. The mobile phase consisted of $10 \mathrm{mM}$ ammonium formate in water (A) and $10 \mathrm{mM}$ ammonium formate in methanol. The flow rate was set at $0.250 \mathrm{ml} /$ min, and the column temperature was $35^{\circ} \mathrm{C}$. The gradient conditions used for the separation of amino acids derivatives were $68 \% \mathrm{~B}$ from 0 to 19 min followed by linear gradient to $93 \%$ B. The column was held at $93 \%$ B from 19.5 to $19.6 \mathrm{~min}$. Then returned to initial conditions 68\% B from $19.6 \mathrm{~min}$ and allowed to equilibrate for $4.4 \mathrm{~min}$. Run-to-run time was $24 \mathrm{~min}$. The injected volume was $5 \mu \mathrm{L}$.

Mass spetrometric conditions as follows: the electrospray ion source (ESI) were operated under positive ion mode; using a nebulizing gas pressure set at $2.8 \mathrm{bar}$, the drying gas flow at $12 \mathrm{~L} / \mathrm{min}$, the drying gas temp at $300^{\circ} \mathrm{C}$. Before each separation run, a calibrant solution of sodium formate was injected. The control of the instrument and the data processing were done using TofControl 3.2 and Data Analysis 4.1 (Bruker Daltonics), respectively.

Quantitative evaluation of amino acids and ratios to creatinine. Quantification of free amino acids was made by reporting to intenal standard HARG. Amino acids concentrations were calculated as formula: $\mathrm{C}=\left(\mathrm{A}_{\mathrm{AA}}{ }^{*} \mathrm{C}_{\mathrm{HARG}}\right) / \mathrm{A}_{\text {HARG }}$ were $C$ represents the concentration of amino acids, $\mathrm{A}_{\mathrm{AA}}$ represents the area of amino acids, $\mathrm{C}_{\text {HARG }}$ represents the concentration of internal standard and $A_{\text {HARG }}$ represents the area of internal standard. The amino acids concentrations were reported to creatinine. The standard curves for creatinine were validated over the concentration range of $0.1-0.0062 \mathrm{mg} / \mathrm{ml}$ from five dilutions. Average correlation coefficient $\left(\mathrm{r}^{2}\right)$ for the curve was 0.95.

Biostatistic interpretation of data. Chromatograms were processed using the Extracted Ion Chromatogram method from Data Analysis 4.1 (Bruker Daltonics) software.

\section{RESULTS AND DISCUSSION}

Chromatographic separation and MS identification of aminoacids using Extracted Ion chromatogram (EIC). Using UPLC-QTOF-ESI(+) MS analysis and a specific Data Analysis software (Extracted Ion chromatogram), 16 derivatized amino acids were separated and identified as adducts of amino acids with propyl chloroformate. Figures $1 A$ and $1 B$ shows the profiles obtained for male and female urine, respectively.

The general fingerprint showed 16 molecules identified (http://www.phenomenex.com/Products/AminoAcidDetail/EZfaast).

The comprehensive list of derivatized amino acids identified by their molecular mass and weight and retention time measured on under the conditions specified above are presented in Table 1. The identification of the amino acids in the chromatograms are given by peak number assigned.

Quantitative analysis. From a total of 27 amino acids, we quantified a number of 16 , out of which 13 were found in both cases (Fig. 2). Amino acids concentrations were expressed in nmol per $\mathrm{g}$ of urinary creatinine.

Male showed higher levels in 10 of the 16 investigated amino acids while only 2 amino acids were higher on female urine profile. Compared with women, men had higher concentration of essential amino acids including histidine $(203.13 \mathrm{nmol} / \mathrm{mg}$ creatinine), lysine (38.53 $\mathrm{nmol} / \mathrm{mg}$ creatinine), phenylalanine (23.67 nmol/mg creatinine) and tryptophan $(60.18 \mathrm{nmol} / \mathrm{mg}$ creatinine). Alanine (18.31 nmol/mg creatinine), cysteine (27.75 $\mathrm{nmol} / \mathrm{mg}$ creatinine) and tyrosine $(118.07 \mathrm{nmol} /$ mg creatinine) were the non-essential amino acids that were higher among the male in this study. Also, a non-proteinogenic amino acid, hydroxyproline (HYP) was significantly higher in man. Glutamine (24.72 nmol/mg creatinine) and valine (19.42 $\mathrm{nmol} / \mathrm{mg}$ creatinine) were the essential amino acids wich showed higher levels in 


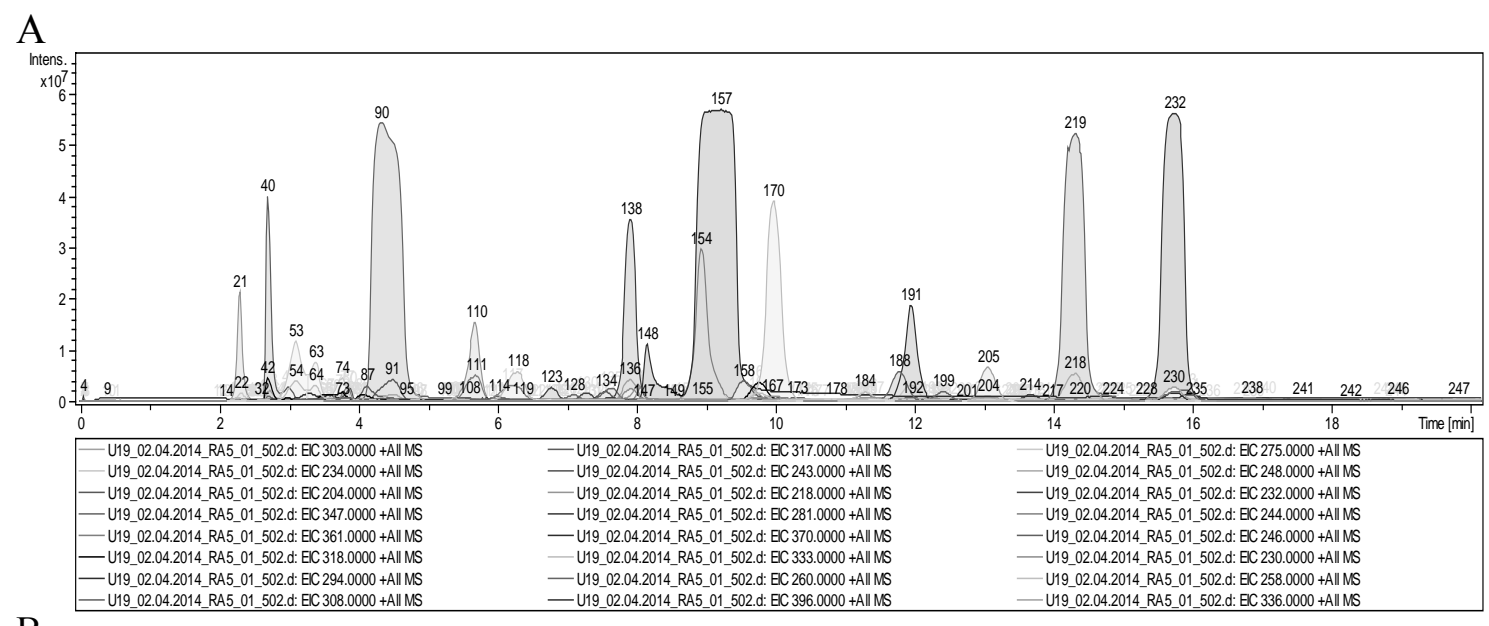

B

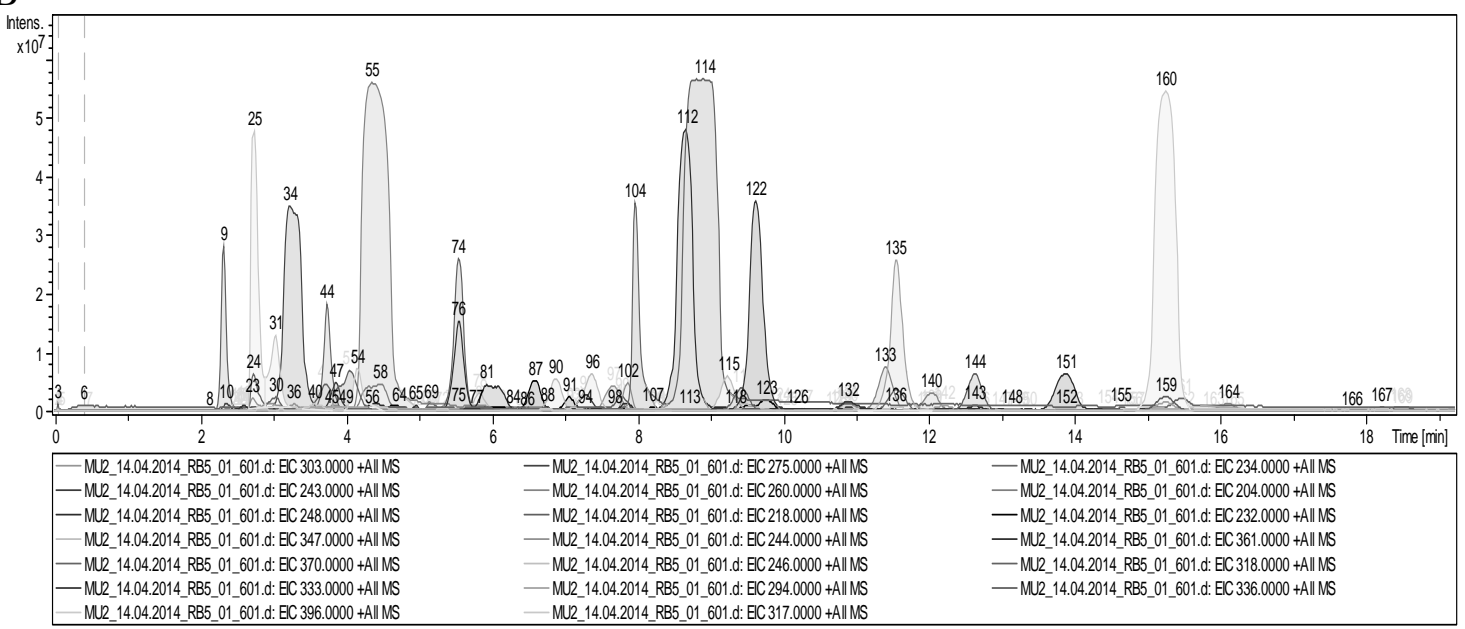

Fig. 1. Extracted ion chromatograms (EIC) for amino acids on male (A) and female (B) urine

Tab. 1. The peak numbers, $\mathrm{m} / \mathrm{z}$ values assigned for derivatized amino acids and molecular weight (MW) amino acids identified by LC/MS and EIC.

\begin{tabular}{|c|c|c|c|c|}
\hline \multicolumn{2}{|c|}{ Peak number } & \multirow{2}{*}{$\begin{array}{c}\mathrm{m} / \mathrm{z} \\
{\left[\mathrm{M}+\mathrm{H}^{+}\right]}\end{array}$} & \multirow{2}{*}{ Amino acids MW } & \multirow{2}{*}{ Amino acid identified } \\
\hline Fig. $1 \mathrm{~A}$ & Fig. 1B & & & \\
\hline 21 & 9 & 218 & 89.1 & Sarcosine \\
\hline 40 & 25 & 317 & 188.2 & Homoarginine-IS \\
\hline 53 & 34 & 275 & 146.2 & Glutamine \\
\hline $\mathrm{NI}$ & 44 & 234 & 105.1 & Serine \\
\hline 90 & 56 & 260 & 131.1 & 4-Hydroxyproline \\
\hline $\mathrm{NI}$ & 65 & 248 & 119.1 & Threonine \\
\hline 110 & 74 & 218 & 89.1 & Alanine \\
\hline 136 & 104 & 281 & 152.2 & Methionine-d3 -IS \\
\hline 142 & 102 & 244 & 115.1 & Proline \\
\hline 154 & 112 & 361 & 146.1 & Lysine \\
\hline 157 & 114 & 370 & 155.1 & Histidine \\
\hline 158 & 115 & 246 & 117.1 & Valine \\
\hline 161 & $\mathrm{NI}$ & 318 & 147.1 & Glutamic acid \\
\hline 170 & 122 & 333 & 204.2 & Tryptophan \\
\hline 188 & 133 & 260 & 131.2 & Leucine \\
\hline 191 & 135 & 294 & 165.2 & Phenylalanine \\
\hline 204 & 144 & 336 & 121.2 & Cysteine \\
\hline 219 & 151 & 308 & 179.2 & Homophenylalanine-IS \\
\hline 232 & 160 & 396 & 181.2 & Tyrosine \\
\hline
\end{tabular}

NI- non-identified; IS-internal standard 


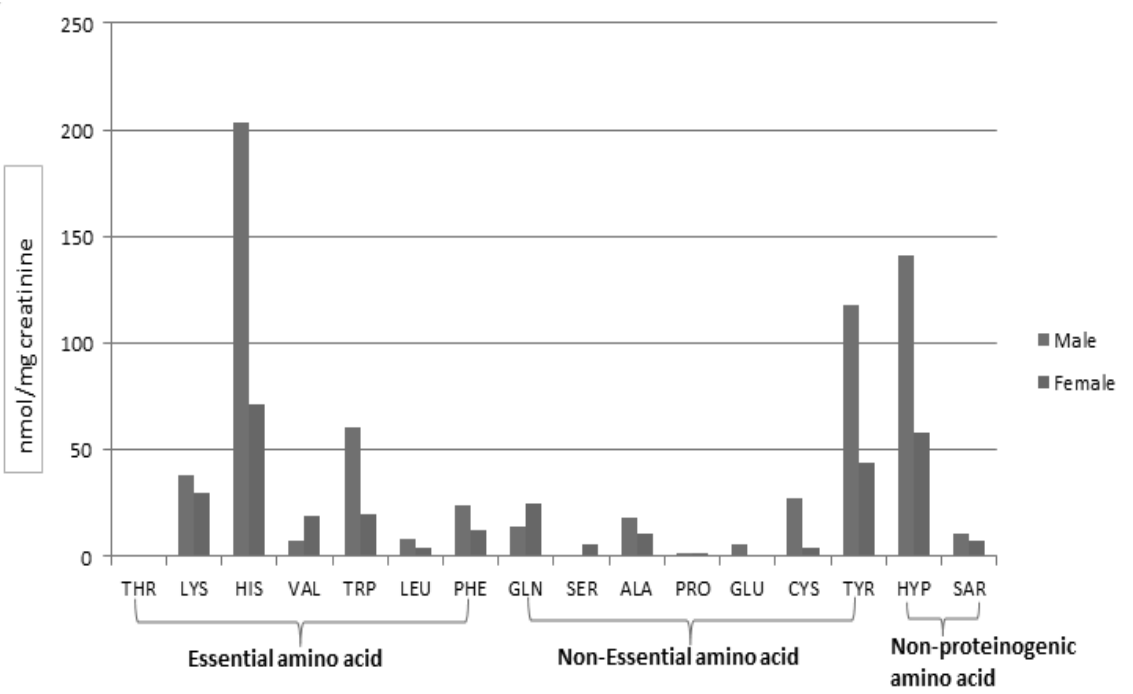

Fig. 2. Comparative concentrations of the 16 identified amino acids, expressed in $\mathrm{nmol} / \mathrm{g}$ creatinine by LC-QTOF-(ESI+)-MS in urine of an healthy male and female.

Tab. 2. Quantitative ratios of essential, non-essential and non-proteinogenic amino acids

\begin{tabular}{lcc}
\cline { 2 - 3 } & Male & Female \\
\hline TFAA (nmol/mg creatinine) & 679.2265 & 313.0746 \\
\hline EAA (\%) & 50.24081 & 50.3984 \\
\hline NEAA (\%) & 27.31762 & 28.80036 \\
\hline NP $(\%)$ & 22.44157 & 20.80124 \\
\hline
\end{tabular}

TFAA-total free amino acid, EAA-essential amino acid, NEAA-non-essential amino acid, NP-non-proteinogenic amino acids.

female. No serine and threonine were detected in male and no glutamic acid in female. Our findings were correlated with other studies (Hassan, 2011, Pitkanen, 2003). Significantly higher levels of histidine and tryptophan were reported in males vs females while the female serum had higher concentration of valine (Caballero et al., 1991). No significant differences in amino acid profile between gender were found in case of proline.

Quantitative ratios of essential, non-esential and non-proteinogenic amino acids from total free amino acids were quantified, as presented in Table 2.

From the total free amino acids around 50\% were essential amino acids, this ratio being similar in male and female. The non-essential amino acids represented $27-28 \%$, while the non-proteinogenic ones represented around 20-22\%. No significant differences were noticed between genders.

Such evaluation provides information about a good nutrition and health, but lower levels can reflect digestion deficiencies (ex. hystidine decrea- se), inadequate stomach acid secretion (lower concentration of tryptophan, phenylalanine and tyrosine) as reported (Bongiovanni et al., 2003).

Disturbances in aminoacid profiles can indicate also pathologic processes like cancer, by modification of cell metabolic pathways and synthesis (Vissers et al., 2005; Slupsky et al., 2010) or cardiovascular diseases (Tang et al., 2010).

\section{CONCLUSION}

Using a rapid UPLC-QTOF-ESI(+)MS method, including apre-column derivatization for amino acids, the comparative profile of male amnd female urine was obtained. By extract ion chromatogram, the quantitative composition of 16 amino acids was evaluated.

The general fingerprint of the two samples was similar, while the concentrations of male amino acids were generally higher (aprox. 2 times) as compared with women, mainly for essential amino acids. The most abundant amino acid was hystidine, two times higher in male than female. 
Also, tyrosine and tryptophan were much higher in man.

Considering the percentages of each subclass of amino acids, the values were not significantly different among gender, the essential amino acids representing around 50\% from the total free amino acids.

The method applied is very useful to identify modifications in the amino acid prodile in different pathologies and metabolic diseases.

\section{REFERENCES}

1. Baniasadi H, Gowda GA, Gu H, Zeng A, Zhuang S, Skill N, Maluccio M, Raftery D (2013). Targeted metabolomic profiling of hepatocellular carcinoma and hepatitis C using LC-MS/MS. Electrophoresis; 34(19): 2910-7.

2. Bhutia YD, Babu E, Prasad PD, Ganapathy V (2014). The amino acid transporter SLC6A14 in cancer and its potential use in chemotherapy. Asian Journal of Pharmaceutical Science I-II.

3. Bongiovanni B, Feinerman J, David MR, Lord RS, Bralley JA (2003). Amino acid profiling. Clinical Guidelines for determination of preferred specimen choice. Townsend Letter for Doctors and Patients: the Examiner of Medical Alternatives 245:38-42

4. Caballero B, Gleason RE, Wurtman RJ (1991). Plasma amino acid concentration in healthy elderly man and women. American Journal of Clinical Nutrition 53:12491252.

5. Grebe S, Ravinder S (2011). LC-MS/MS in the clinical laboratory -Where to from here?. Clinical Biochemist Reviews, 32: 5-31.

6. Hassan DA, Ibrahim A, Musa AB, Abukunna FE, Mohamed NM, Ahmed SIS, Hassan HM and Hussein AR (2011). Normal levels of plasma amino acids among sudanese adults: the effect of gender. Journal of Medical Sciences 11:246-249.

7. Kami K, Fujimori T, Sato H, Sato M, Yamamoto H, Ohashi Y, Sugiyama N, Ishihama Y, Onozuka H, Ochiai A, Esumi H, Soga T, Tomita M (2013). Metabolomic profiling of lung and prostate tumor tissues by capillary electrophoresis time-of-flight mass spectrometry. Metabolomics 9: 444453.

8. Kasture VS, Musmade D, Vakte M, Sonawane S, Patil P (2012). Metabolomics: Current technology and future trends International Journal of Research and development in Pharmacy and Life Sciences 2(1): 206-217.

9. Lai HS, Lee JC, Lee PH, Wang ST, Chen WJ (2005). Plasma free amino acid profile in cancer pacients. Cancer Biology 15:267-276.
10. Meesters RJW, Wolfe RR, Deutz NEP (2008). Application of liquid chromatography-tandem mass spectrometry (LCMS/MS) for the analysis of stable isotope enrichments of phenylalanine and tyrosine. Journal of Chromatography B 877:43-49.

11. Miyagi Y, Igashiyama $M$, Gochi A, Akaike M, Ishikawa T, Miura T, Saruki N, Bando E, Kimura H, Imamura F, Moriyama M, Ikeda I, Chiba A, Oshita F, Imaizumi A, Yamamoto $H$, Miyano $H$, Horimoto $K$, Tochikubo 0 , Mitsushima T, Yamakado M, Okamoto N (2011). Plasma free amino acid profiling of five types of cancer patients and its application for early detection. Plos One, 6(9): e24143.

12. Okamoto N, Miyagi Y, Chiba A, Akaike M, Shiozawa M, Imaizumi A, Yamamoto H, Ando T, Yamakado M, Tochikubo 0 (2009). Diagnostic modeling with differences in plasma amino acid profiles between non-cachectic colorectal/breast cancer patients and healthy individuals. International Journal of Medicine and Medical Sciences 1(1): 001-008.

13. Pitkanen HT, Oja SS, Kemppainen K, Seppa MJ, Mero AA (2003). Serum amino acid concentrations in aging men and women. Amino acids 24: 413-421.

14. Slupsky CM, Steed H, Wells TH, Dabbs K, Schepansky A, Capstick V, Faught W, Sawyer MB (2010). Urine metabolite analysis offers potential early diagnosis of ovarian and breast cancers, Clin. Cancer Res 1; 16(23): 5835-41.

15. Tang WH, Wang Z, Cho L, Brennan DM, Hazen SL (2009). Diminished global arginine bioavailability and increased arginine catabolism as metabolic profile of increased cardiovascular risk. Journal of the American College of Cardiology 53:2061-2067.

16. Ubhi BK, Davenport PW, Welch M, Riley J, Griffin JL, Connor SC (2013). Analysis of chloroformate-derivatised amino acids, dipeptides and polyamines by LC-MS/MS. Journal of chromatography B 934:79-88.

17. Vissers YLJ, Dejong HC, Luiking Y, Fearon KCH, Meyenfeldt MF, Deutz NEP (2005). Plasma arginine concentrations are reduced in cancer patients: evidence for arginine deficiency? The American Journal of Clinical Nutrition 81:1142-6.

18. Wang C, Zhu H, Pi Z, Song F, Liu Z, Liu S (2013). Clasification of type 2 diabetes rats based on urine amino acids metabolic profiling by liquid chromatography coupled with tandem mass spectrometry. Journal of Chromatography B 935:26-31.

19. Wilson SF, James CA, Zhu X, Davis MT, Rose MJ (2011). Development of a method for the determination of glycine in human cerebrospinale fluid using pre-column derivatization and LC-MS/MS. Journal of Pharmaceutical and Biomedical Analysis 56: 315-323.

20. ht tp : / / w w w.phenomenex.com / Products / AminoAcidDetail/EZfaast 